\title{
Comment on "Coronavirus disease 2019 pandemic and anxiety: a longitudinal study in 287 Brazilians"
}

\author{
Ran $\mathrm{Hou}^{1} \odot$, Tinghui $\mathrm{Li}^{1 *}$
}

Dear Editor,

We are pleased to read the interesting paper published by Souza et al. ${ }^{1}$. Their findings revealed that a high prevalence of anxiety lowered after 8 weeks. Anxiety was worse in women, in youngsters, and in those who worked or studied in the area of health. Although this study is of great significance for the prevention of anxiety during the coronavirus disease 2019 (COVID-19) pandemic, I believe that some issues should be raised.

To begin with, the main problem of this article is the lack of describing the general demography in detail, such as gender, age, place of residence, economic level, educational level, occupation, and so on. Later, the results showed that women and young people were more easily to become anxious; however, most of the participants of this research were women and young people, which might cause selection bias.

In addition, the research only found that women and young people were more easily to become anxious, but further discussion on the influencing factors of anxiety among the women and young people should be described. Finding out the risk factors of anxiety among women and young people has an important guide value to the intervention of the COVID-19.

Finally, another concern is that the definition of anxiety and the range of age for young people should be provided in the methods part of this study. The maximum score of instruments was 63 points, and the participants were classified according to the degrees of anxiety: minimum: $0-10$, mild: $11-19$, moderate: $20-30$, and severe: $31-63$. Meanwhile, the cutoff value of defining anxiety should be offered in this study.

\section{AUTHORS' CONTRIBUTIONS}

RH: Conceptualization, Data curation, Formal Analysis, Writing - original draft, Writing - review \& editing. TL: Conceptualization, Data curation, Formal Analysis.

\section{REFERENCE}

1. Souza ACM, Manoel AZ, Manoel PZ, Weiler RA, Kimura RNY, Skare TL. Coronavirus disease 2019 pandemic and anxiety: a longitudinal study in 287 Brazilians. Rev Assoc Med Bras (1992). 2021;67(4):516-21. https://doi.org/10.1590/1806-9282.20200881 